\title{
Docentes resilientes. Elementos centrales en el programa universitario de tutoría
}

\author{
Resilient teachers. Central elements in the University tutoring program
}

\author{
Erick Cajigal Molina
}

\begin{abstract}
RESUMEN
La resiliencia se puede desarrollar en los espacios del programa de tutoría universitaria e incide en la reprobación y deserción de los estudiantes. Los docentes tienen mayores posibilidades de participar en el desarrollo o mejora de la resiliencia de los estudiantes al ser resilientes. De ahí que se presenta una investigación realizada con el objetivo de conocer el estado que guardan los aspectos que componen la resiliencia de un grupo de docentes de la Universidad Autónoma del Carmen, México, en el año 2020. Es un estudio cuantitativo de tipo correlacional. Se utiliza el instrumento encuesta para tutores de educación, el cual fue sometido a la prueba Alfa de Cronbach resultando con alta confiabilidad. La información fue tratada en el programa SPSS, se realizó la descripción de los ítems por medio de frecuencias y se hicieron cruces de variables con la prueba estadística Chi-cuadrado de Pearson. Los resultados dan la oportunidad de señalar que los participantes poseen una resiliencia aceptable, lo cual les da posibilidades de participar de manera pertinente en el desarrollo de la resiliencia en sus estudiantes desde el programa de tutoría. No obstante, se identifican áreas de oportunidad en los docentes que les impiden posicionarse en la resiliencia deseable y que deben atenderse.

Palabras clave: deserción escolar, docentes, resiliencia, tutoría universitaria.
\end{abstract}

\begin{abstract}
Resilience can be developed in the universities spaces designated for tutoring program, and it affects the students' failure and desertion rate. Professors are more likely to participate in developing or enhancing students' resilience by being resilient themselves. Hence, an investigation carried out with the objective of knowing the state of the aspects that make up the resilience of a group of Professors from the Universidad Autónoma del Carmen, Mexico, in 2020. It is a correlational quantitative study. The instrument used is the survey for education tutors, which was subjected to Cronbach's Alpha test, resulting in high reliability. The information was processed in the SPSS software, the description of the items was carried out by means of frequencies and variables were crossed with Pearson's Chisquare statistical test. The results show that the participants have acceptable resilience, which gives them the opportunity to participate in a relevant way in the development of their students resilience. However, areas of opportunity are identified in teachers that prevent them from positioning themselves in the desirable standards and that must be addressed.
\end{abstract}

Keywords: school dropout, teachers, resilience, university tutoring. 


\section{INTRODUCCIÓN}

Los problemas inacabables e inseparables que preocupan a las universidades, como la reprobación y la deserción escolar, han sido en gran parte abordados por el programa de tutoría. Se considera que las tutorías universitarias son una respuesta de las instituciones de educación superior para mejorar el desempeño de los estudiantes en riesgo (Guerra-Martín, Lima-Serrano y Lima-Rodríguez, 2017).

Es necesario considerar a las tutorías como un programa integral en el cual se trabajan aspectos académicos, intelectuales, psicológicos, emocionales y personales de los estudiantes; tal como lo hacen notar Narro y Arredondo (2013, p. 138), la tutoría es "una intervención docente en el proceso educativo de carácter intencionado (...) para apoyarlo y facilitarle el proceso de construcción de aprendizajes de diverso tipo: cognitivos, afectivos, socioculturales y existenciales". La tutoría abre mayores posibilidades a los universitarios de formarse de manera exitosa, debido a que incide en la reprobación, la deserción y el desempeño escolar (Faroa, 2017).

La tutoría, como programa universitario de formación integral, tiene diversos puntos de encuentro con las estrategias que se consideran en el desarrollo de la resiliencia. Las personas a lo largo de su desarrollo experimentan diversas adversidades, y la teoría de la resiliencia da comprensión sobre cómo hacen frente a los problemas y dificultades de la vida (Taormina, 2015). Por su parte, Cruz-Flores, Cheyhaybar y Abreu (2011) señalan que la tutoría ha cobrado un peso relevante en problemáticas de las instituciones de educación superior como son la reprobación, el rezago y la deserción, debido a que el acompañamiento y seguimiento de los estudiantes a lo largo de su trayectoria escolar puede disminuir tales problemáticas.

Se han registrado esfuerzos en instituciones de educación superior en torno a la actualización de estrategias en el programa de tutoría universitaria, a partir del desarrollo de la resiliencia. Por ejemplo, en la investigación de Cajigal, Hernández-Marín, Yon y Arias (2020) se muestra el inicio de una estrategia que pretende reducir el porcentaje de reprobación y deserción escolar, además de incrementar el desempeño escolar de estudiantes con el desarrollo de la resiliencia desde el programa de tutoría. En la mencionada investigación se identifica que la mayoría de los estudiantes se posiciona en una etapa de resiliencia aceptable, no obstante el fin último de la estrategia

Erick Cajigal Molina. Profesor-investigador en la Facultad de Ciencias Educativas de la Universidad Autónoma del Carmen, Campeche, México. Es licenciado en Intervención Educativa por la Universidad Pedagógica Nacional, además es maestro y doctor en Investigación Educativa por la Universidad Veracruzana. Tiene los reconocimientos al perfil ProdeP y del Sistema Nacional de Investigadores. Colabora en el Cuerpo Académico de Investigación en Humanidades. Entre sus publicaciones recientes se encuentran "Resiliencia de tutorados. Un caso de la Facultad de Ciencias Educativas de la Universidad Autónoma del Carmen, México" y "Situación de los posgrados en Campeche. Estado actual ante los parámetros del ConAcyT". Correo electrónico: cajigal1983@hotmail.com. ID: https://orcid.org/0000-0002-6633-5116. 
es posicionar a los estudiantes en la etapa deseable, de tal forma que se incrementen las posibilidades para disminuir el porcentaje de reprobación y deserción escolar.

Para ello, un elemento fundamental en el desarrollo de la resiliencia es la figura de un adulto significativo en los universitarios. En concreto, esta figura se refiere a una persona que provee modelos de conducta y de resolución de problemas pertinentes, es aquel individuo que crea un ambiente de respeto, confianza, colaboración y comunicación, además es estimada y estima a las personas con las que interactúa (Melillo, 2001). Se piensa que la figura de adulto significativo para desarrollar la resiliencia puede ser asumida por el docente universitario en su rol de tutor, sin embargo para participar de manera adecuada se espera que sean resilientes; "sería poco realista pretender que los alumnos fueran resilientes si sus docentes no lo son" (Henderson y Milstein, 2003, p. 57).

Por lo tanto, la presente investigación tuvo como objetivo conocer el estado que guarda cada uno de los aspectos que componen la resiliencia de los docentes de la Licenciatura en Educación (LE) de la Universidad Autónoma del Carmen (UNACAR), a fin de proponer acciones que contribuyan en la mejora de los factores que resulten negativos. ${ }^{1}$

Es preciso indicar que esta investigación se suscribe a los estudios que cambian la forma en que se percibe a las personas, de un modelo de riesgo basado en las necesidades a un modelo de prevención y de promoción de las capacidades que poseen junto con los recursos que disponen a su alrededor (Fontaines y Urdaneta, 2009).

\section{Resiliencia}

La resiliencia como teoría surge para dar comprensión a las diferentes formas de hacer frente a las adversidades, pues es claro que "mientras algunas personas sucumben ante circunstancias e imprevistos generando desequilibrio y trastornos a diversos niveles, otras se desarrollan exitosamente a pesar de la adversidad" (Fontaines y Urdaneta, 2009, p. 165).

El término de resiliencia está en boga y se ha adoptado para referirse a "invulnerable", "invencible" y "resistente", además se ha reconocido como un proceso de esfuerzo y lucha (Noriega, Angulo y Angulo, 2015). En este sentido, la resiliencia se concibe desde dos caminos: el genetista, que refiere a las personas que muestran características resilientes innatas, y el de desarrollo, que concibe la posibilidad de construir resiliencia en las personas (Melillo, 2001). En investigaciones científicas el término se ha descrito desde múltiples perspectivas, destacando tres: superar la adversidad (recuperarse), preparación para las adversidades venideras (orientación preventiva y proactiva) y aprender de las adversidades (transformarse) (OrtegaGonzález y Mijares, 2018).

1 La UNACAR es una institución de educación superior de carácter público. Sus campus se ubican en el estado de Campeche, México. 
Se concuerda con la psicología positiva el pensar que no todos los sujetos al enfrentarse a situaciones problemáticas desarrollarán enfermedades, traumas o trastornos, sino que la mayoría posee una capacidad natural de superar dichas situaciones, aprendiendo de estas, incluso beneficiándose (Díaz-Sánchez y Barra, 2017). Es así como algunas personas después de las adversidades no solo resistieron o se recuperaron, en definitiva, también aprendieron y se transformaron.

De ahí que se considera que la resiliencia es una capacidad emocional que surge para resistir, recuperarse o transformarse ante las adversidades, esta se nutre con factores de orden social, ambiental, afectivos, carácter, cognitivos y de salud (física y mental). Cabe decir que al pensarse la resiliencia como capacidades, se asume que estas se pueden desarrollar o mejorar, no obstante también pueden mostrar un cambio negativo. Dicho diferente, la resiliencia no es una condición permanente, esta evoluciona, cambia conforme nos desarrollamos y está en función del equilibrio resultante de los factores protectores y las adversidades (Aguaded y Almeida, 2016).

Los factores protectores hacen referencia a aspectos de orden interno y externo en las personas, como por ejemplo los ya mencionados: autoestima, confianza, personalidad, apoyo, figuras significativas, cohesión familiar, ambiente en la escuela, así como salud física y emocional. En un sentido figurado, cuando las personas experimentan una adversidad, los factores protectores entran en una batalla contra las adversidades, también llamados factores de estrés; si los primeros están fortalecidos tendrán más posibilidades de resultar victoriosos, demostrando ser resilientes.

La investigación científica desde la psicología positiva busca comprender la mencionada batalla (interacción) de factores a través de "los procesos que subyacen a pensamientos, actitudes y emociones positivas del ser humano" (Noriega, Angulo y Angulo, 2015, p. 47). La información que se obtiene permite establecer acciones que incidan en cada uno de los factores protectores que resulten negativos en las personas, y así desarrollar o mejorar la resiliencia.

En suma, la resiliencia se refiere a las capacidades que la personas poseen para hacer frente a las situaciones adversas. Las investigaciones en este tema permiten comprender las situaciones y factores que hacen a las personas, grupos o comunidades vulnerables, al mismo tiempo identifican las condiciones que los encaminan a un desarrollo físico y psicológico, saludable y equilibrado (Aguaded y Almeida, 2016). De ahí que, para esta investigación, es preciso definir cada uno de los factores que se consideran dentro de la resiliencia de los docentes universitarios, indagar sobre el estado que guardan y generar propuestas que fortalezcan a los que resulten en áreas de oportunidad.

\section{Características de un docente resiliente}

Se concuerda con Fontaines y Urdaneta (2009) al señalar que existe un rescate de la condición humana para acceder a una vida productiva, y ha sido adoptado por algunas 
universidades latinoamericanas en los últimos años. Este rescate considera que la simple adquisición de recursos humanos se ha visto infructuosa frente a la gestión del capital humano, la cual coloca a sus colaboradores como un recurso valioso ante casos de conciliación y riesgo.

Pensar en la gestión del capital humano en las instituciones educativas nos obliga a concebir, en este caso, a los educadores como personas que crecen e interactúan con grupos sociales internos y externos a sus espacios de trabajo, que lo alteran, lo modifican, y a su vez ellos son capaces de incidir recíprocamente (Aguaded y Almeida, 2016). Todo esto permea su actuación frente a sus estudiantes y tutorados, por esta razón se ha mencionado que "es preciso, pues, desarrollar la motivación y la resiliencia en el docente; esto posibilitaría crear un maestro optimista, con la convicción [de] que, incluso en las condiciones actuales de la escuela, se pueden obtener resultados positivos en el alumno" (Flores-Espinoza, 2017, p. 151).

La universidad impacta de manera positiva o negativa en los factores que se consideran en la resiliencia de los docentes, y esto a su vez permea su práctica. La docencia y la tutoría que se llevan a cabo con conocimientos, actitud pertinente, templanza y convicción, demuestran factores resilientes estables. En esta investigación se considera al docente y tutor como:

[Un] vehículo, como acompañante, como escudo protector, como fuente de resiliencia, necesitaría conectarse consigo mismo, con sus temas de vida, con sus posibilidades realistas y con su propia resiliencia para poder acompañar y dar sentido a su actuar, que va más allá de la transmisión de información [Acevedo y Restrepo, 2012, p. 308].

Por lo tanto, la atención hacia los docentes es de vital importancia en las universidades, y esta no solo debe de enfocarse en capacitaciones académicas sino también es menester considerar aspectos en relación con lo ambiental, lo afectivo y lo personal, así como la templanza y la salud (física y emocional). "La clave fundamental es que la escuela sea capaz de ofrecer los reforzadores de los factores protectores en la vida de los estudiantes y de los docentes" (Noriega, Angulo y Angulo, 2015, p. 43).

Se encuentran elementos puntuales que hacen referencia a las características de un docente resiliente:

- Posee capacidad de introspección, independencia, capacidad de relacionarse, iniciativa, humor, creatividad y moralidad (Fontaines y Urdaneta, 2009).

- Invierte en su formación, tiene conocimientos de sí mismo, expresa de manera coherente sus valores, conocimientos y creencias, domina las situaciones con seguridad y control, no tiene miedo de intervenir, no muestra estrés; además se ve constantemente acompañado por sus compañeros pues encuentra entendimiento en ellos, muestra motivación en las actividades que comparte con sus colegas (Aguaded y Almeida, 2016).

- Es frecuentemente referido por sus alumnos como el maestro especial o consentido y se convierte en un modelo de conducta positivo para ellos; también 
se muestra exitoso y tiende a mantener estándares académicos altos (Noriega, Angulo y Angulo, 2015).

- Muestra autonomía, pero también trabaja en equipo; considera que los recursos con los que trabaja son suficientes, externa sus opiniones y experiencias para mejorar su labor, crea espacios para abordar temas o problemas ajenos a sus cursos. También busca proyectos educativos para desarrollar la resiliencia de los estudiantes (Flores-Espinoza, 2017).

- Muestra destrezas y habilidades que pone en práctica en situaciones no rutinarias, además de conocimientos propios de su formación superior. Además otorga identidad profesional, así como un conjunto de competencias profesionales en sus estudiantes (Jadue, Galindo y Navarro, 2005).

- Se asume como una figura de resiliencia y cambio. Crea ambientes donde se acepta la diversidad y se comprenden las situaciones difíciles que atraviesan los estudiantes. Asimismo mantiene una mirada de optimismo hacia los estudiantes (Acevedo y Restrepo, 2012).

Esta investigación planteó conocer el estado que guardan los aspectos que componen la resiliencia de los docentes de la LE, con la intención de generar propuestas para aquellos que resulten como áreas de oportunidad. Por consiguiente, se describen los factores estudiados y la ruta metodológica adoptada.

\section{Metodología}

La metodología en esta investigación fue cuantitativa de tipo correlacional. Se trabajó con la técnica de la encuesta para recolectar la información. Esta contó con escala Likert, distribución de respuesta de 25\%, y fue sometida a la prueba de confiabilidad Alfa de Cronbach. Cabe decir que, previo a la aplicación, el instrumento fue sometido a un pilotaje en el mes de mayo del 2020, con docentes del Centro de Estudios Superiores Isla del Carmen (ubicado en Ciudad del Carmen, Campeche, México), en donde participaron seis docentes, dando cuenta de que las afirmaciones eran claras y que el tiempo promedio para responder era de diez minutos. Con los resultados del pilotaje, el instrumento fue sometido a la prueba de confiabilidad Alfa de Cronbach, para lo cual se identificaron los ítems invertidos $(9,11,16,18,19$ y 22) y se transformaron (se cambió su valoración en el SPSS). En la prueba se obtuvo un valor de 0.89, lo que permite señalar que es un instrumento confiable (Taber, 2017).

De las cuatro opciones de respuesta, dos se consideraron positivas (muy de acuerdo y de acuerdo) y dos negativas (muy en desacuerdo y desacuerdo). La captura de información, así como la aplicación de las pruebas estadísticas para su análisis, se realizó en el programa IBM Statistical Package for the Social Sciences (SPSS), versión 22. En este sentido, en el análisis se realizó una descripción por medio de frecuencias en cada 
ítem, se realizaron tablas de contingencia con la prueba Chi cuadrada de Pearson para determinar la correlación entre las variables utilizadas y se ofrecieron resultados por dimensión, así como de manera holística.

Se trabajó con una muestra representativa de la población de los docentes que laboran en el programa de LE, los cuales también son tutores de estudiantes de este mismo programa educativo. El tamaño de la muestra coincide con una confiabilidad del estudio de $90 \%$. El muestreo fue probabilístico, por sujetos voluntarios. En este tenor, de los 12 docentes que laboran en el programa educativo, la muestra se conformó por 11 que atendieron la invitación a participar en el estudio; solo un docente no participa pues existe un conflicto de interés: es autor del presente documento.

Por otro lado, las personas responden a las experiencias y adversidades "como resultado de una multitud de aspectos, internos y externos" (Aguaded y Almeida, 2016, p. 169). De ahí que los factores considerados estuvieron divididos en dos categorías: interna y externa, cada una a su vez estuvo integrada por variables e indicadores que permitieron su estudio.

En cuanto a la categoría interna se consideraron las variables:

- Personalidad. Esta variable se estudió con base en la propuesta de Henderson (2001) sobre el factor Yo, que incorpora cuatro elementos: Yo tengo (apoyo), Yo soy (autoestima), Yo estoy (autoestima) y Yo puedo (confianza y actitud).

- Responsabilidad. Los educadores que están conscientes de su responsabilidad en sus actividades dentro y fuera de las instituciones educativas fomentan en sí mismos la resiliencia (Noriega, Angulo y Angulo, 2015).

- Habilidad de resolución de problemas. Aguaded y Almeida (2016) señalan que un educador que resuelve sus problemas con control y seguridad muestra conocimiento de sí mismo. Los retos y las nuevas situaciones con las que se enfrentan los educadores ocasionan estrés y depresión, aumentando su incapacidad de controlar las situaciones cotidianas.

- Condición de salud y física. El estado que guarda su salud y la condición física contribuye o no a que las personas puedan hacer frente a las adversidades (Richardson, Neiger, Jensen y Kumpfer, 1990).

- Habilidades de comunicación y empatía. La facilidad para comunicarse y la capacidad de participar afectivamente en una realidad ajena son atributos propios de las personas resilientes (Aguaded y Almeida, 2016).

- Expresiones de afecto. Al expresar su reconocimiento a sus estudiantes, colegas y superiores ante una acción que causa satisfacción y merece ser recompensada, se muestra resiliencia (Henderson y Milstein, 2003).

- Expectativas. Cuando los docentes poseen elevadas esperanzas y ven potencial en sus estudiantes, muestran motivación y gusto por su trabajo (Jadue, 2005), elementos que abonan a la resiliencia. 
- Humor. Entre las características de un docente resiliente está el humor (Fontaines y Urdaneta, 2009). Se coincide con Morán-Astorga, Finez-Silva, Menezes dos Anjos, Pérez-Lancho, Urchaga-Litago y Vallejo-Pérez (2019) al señalar que el humor significa hacer bromas o reírse de las situaciones adversas y es una estrategia que aumenta la resiliencia.

Sobre la categoría externa, las variables que se consideraron son:

- Ambiente en la institución educativa. Con base en Henderson y Milstein (2003), se establecieron cinco elementos en esta variable: 1) Vinculos prosociales, que refiere a la vida profesional de los docentes, no solo en compañía exclusiva de sus alumnos sino también con la de sus pares, en actividades a favor de la institución; 2) Limites claros y firmes, este elemento habla sobre la aplicación de reglas y políticas de una manera justa entre todos los docentes; los docentes se muestran seguros cuando los límites son claros y firmes; 3) Capacitación constante, para enfrentarse a los desafíos que se presentan dentro de los espacios educativos, los docentes necesitan desarrollo profesional en función de los cambios sociales y los desarrollos tecnológicos; 4) Expresiones de afecto y apoyo, al recibir el reconocimiento de sus estudiantes, colegas y superiores ante una acción satisfactoria, se abona a la resiliencia; 5) Oportunidades de participación, cada docente tiene habilidades y conocimientos ajenos a su quehacer cotidiano y que puede ofrecer a las instituciones educativas; dar la oportunidad de aportar tales habilidades y conocimientos es promover la resiliencia.

- Comunicación-cooperación familiar. La cohesión familiar es uno de los factores con mayor peso en el desarrollo de la resiliencia (Henderson, 2001); ante una adversidad, la comunicación y cooperación en las familias disminuyen el desánimo y fomentan la movilización de factores protectores.

- Persona(s) significativa(s). La resiliencia tiene mayores posibilidades de desarrollarse cuando existe un vínculo significativo o lazo emocional con algún miembro de su círculo social, el cual proyecte modelos adecuados de resolución de problemas y apoyo directo durante las adversidades (Melillo, 2001).

Como se mencionó, la técnica aplicada por esta investigación fue la encuesta. Cabe resaltar que esta es una de las técnicas que se utilizan con mayor frecuencia en la investigación social cuantitativa, debido a que se obtienen medidas sistemáticas sobre los conceptos que derivan de una problemática de investigación (López-Roldán, 2016). Por todo esto, los resultados globales de la encuesta se interpretaron de la siguiente manera: $60 \%$ de los ítems o menos en positivo, no-resilientes; de $61 \%$ a $74 \%$, resiliencia moderada; de $75 \%$ a $88 \%$, resiliencia aceptable, y de $89 \%$ a 100\%, resiliencia deseable. En la tabla 1 se concentran las variables y los ítems que conformaron el instrumento encuesta para tutores de educación. 
Tabla 1. Categorías, variables e ítems del estudio.

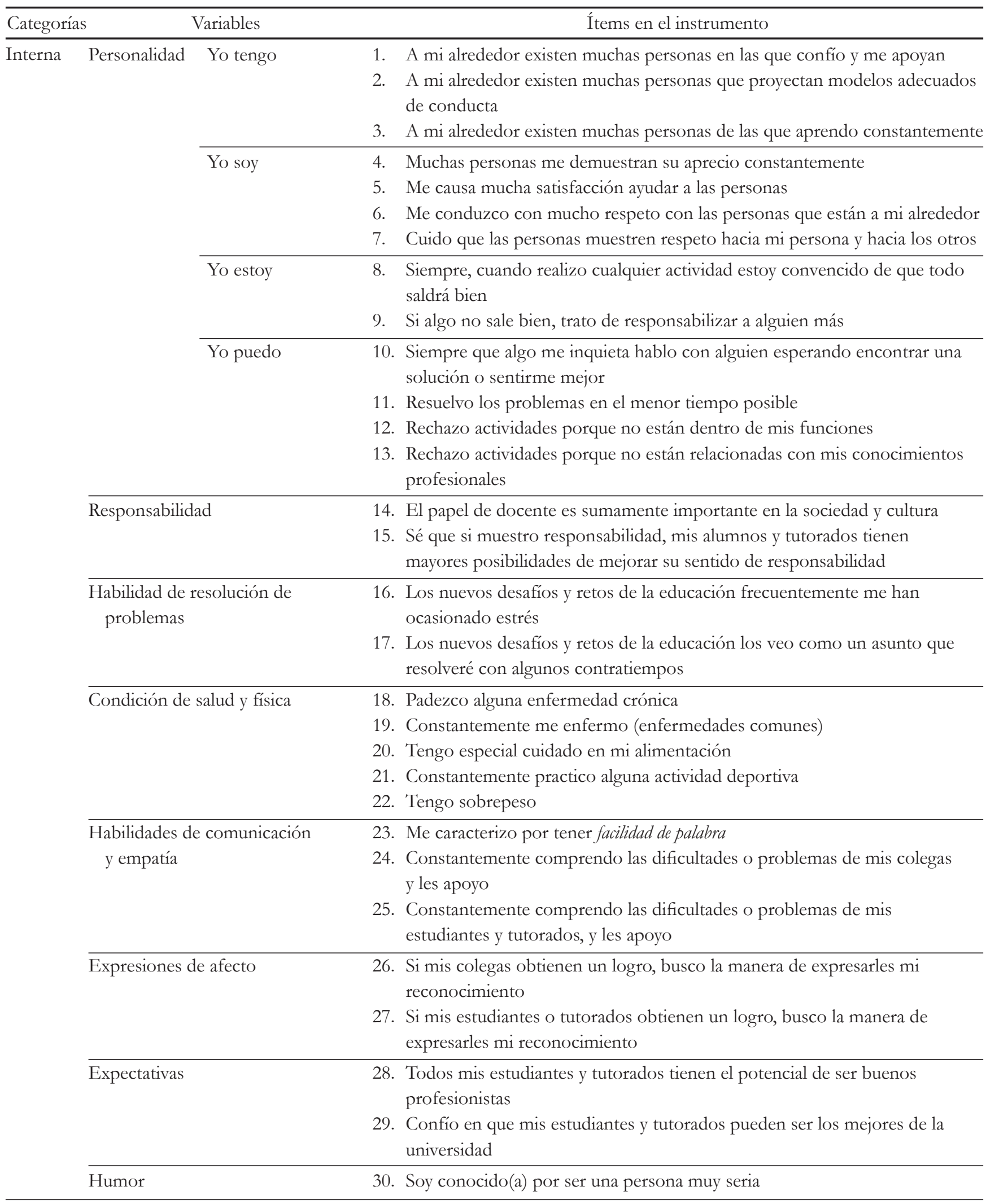


Tabla 1. Categorías, variables e ítems del estudio.

\begin{tabular}{|c|c|c|c|}
\hline \multicolumn{2}{|c|}{ Categorías } & \multirow{2}{*}{$\frac{\text { Variables }}{\text { Vínculos prosociales }}$} & \multirow{2}{*}{$\begin{array}{l}\text { Ítems en el instrumento } \\
\text { 31. Frecuentemente emprendo proyectos o actividades a favor de los } \\
\text { estudiantes y tutorados en colaboración con mis colegas }\end{array}$} \\
\hline \multirow[t]{7}{*}{ Externa } & \multirow[t]{5}{*}{$\begin{array}{l}\text { Ambiente } \\
\text { en la } \\
\text { institución } \\
\text { educativa }\end{array}$} & & \\
\hline & & $\begin{array}{l}\text { Límites claros y } \\
\text { firmes }\end{array}$ & $\begin{array}{l}\text { 33. Las reglas de la universidad se aplican de manera justa y sin distinción para } \\
\text { todos los trabajadores }\end{array}$ \\
\hline & & $\begin{array}{l}\text { Capacitación } \\
\text { constante }\end{array}$ & $\begin{array}{l}\text { 34. Frecuentemente encuentro capacitaciones que mejoran mi quehacer } \\
\text { profesional }\end{array}$ \\
\hline & & $\begin{array}{l}\text { Expresiones de } \\
\text { afecto y apoyo }\end{array}$ & $\begin{array}{l}\text { 35. Las expresiones de aprecio de mis estudiantes, colegas y/o superiores, } \\
\text { son frecuentes } \\
\text { 36. Me siento muy estimado en mi lugar de trabajo }\end{array}$ \\
\hline & & $\begin{array}{l}\text { Oportunidades } \\
\text { de participación }\end{array}$ & $\begin{array}{l}\text { 37. He tenido la oportunidad de proponer y guiar proyectos que están fuera } \\
\text { de la malla curricular, pero tuvieron un beneficio para los estudiantes y } \\
\text { tutorados }\end{array}$ \\
\hline & \multicolumn{2}{|c|}{$\begin{array}{l}\text { Comunicación-cooperación } \\
\text { familiar }\end{array}$} & $\begin{array}{l}\text { 38. Mi familia tiene un nivel alto de comunicación } \\
\text { 39. En mi familia existe un alto nivel de cooperación en las actividades cotidianas } \\
\text { 40. Considero que mi familia es de las que tiene un nivel alto de unión } \\
\text { 41. Si yo viviera una adversidad mi familia estaría muy involucrada para que yo } \\
\text { saliera adelante }\end{array}$ \\
\hline & \multicolumn{2}{|c|}{ Persona(s) significativa(s) } & $\begin{array}{l}\text { 42. Tengo a una persona, muy cercana, que me proyecta modelos adecuados } \\
\text { de conducta y de resolución de problemas }\end{array}$ \\
\hline
\end{tabular}

Fuente: Elaboración propia.

En la tabla 1 se muestran las categorías, variables e ítems que se utilizaron en esta investigación. Los ítems del instrumento se establecen a partir de la literatura: los que corresponden a las variables Personalidad, Condiciones de saludy física, Humor, Ambiente en la institución educativa, Comunicación-cooperación familiar y Persona(s) significativa(s), se desprenden de la investigación de Cajigal, Maldonado y González-Gaudiano (2017); los ítems de la variable Responsabilidad surgen del estudio de Noriega, Angulo y Angulo (2015); los referentes a las variables Habilidad de resolución de problemas y Habilidades de comunicación y empatía se consideran del estudio de Aguaded y Almeida (2016); los ítems sobre las variables Expresiones de afecto y Expectativas se desprenden de Henderson y Milstein (2003) y Jadue, Galindo y Navarro (2005), respectivamente.

En cuanto a la aplicación del instrumento, que se realizó en el mes de junio del 2020, en primer lugar se solicitó el permiso de la directora de la Facultad para la actividad; posteriormente los docentes recibieron en su correo la solicitud de participar en el estudio, al aceptar, se les hacía llegar por el mismo medio la encuesta; finalmente regresaban el documento respondido. La confiabilidad del instrumento también fue positiva en el trabajo de campo, resultando en 0.82 . 


\section{RESUlTADOS Y DISCUSIÓN}

Fueron 11 los docentes participantes, siete mujeres (63.6\%) y cuatro hombres (36.4\%), con una edad que osciló entre 40 y 57 años, con un promedio de 46.1 años. La antigüedad como docentes universitarios fluctuó entre los 10 y 32 años; 18.09 años en promedio. En la tabla 2 se presentan los ítems que corresponden a cada variable y categoría.

En la tabla 2 se concentran los resultados obtenidos por el instrumento encuesta para tutores de educación. La mayor parte de los ítems en este estudio de la resiliencia se posiciona de forma positiva (85.8\% de los ítems), es decir que los docentes participantes poseen resiliencia aceptable. Visto diferente, de los 42 ítems son solo seis (14.2\%) los que presentan un posicionamiento negativo en la mayoría de los docentes, estos son: $11,12,13,16,19$ y 30 . Otros seis tienen áreas de oportunidad con al menos el $25 \%$ de los participantes (ítems 17, 18, 20, 21, 22 y 33); el resto obtuvo un posicionamiento positivo superior al $81.8 \%$.

De la categoría Interna, en la variable Personalidad, el factor Yo puedo es el que más ítems negativos presenta $(11,12$ y 13$)$. No resolver los problemas en el menor tiempo posible y rechazar otras actividades por no estar dentro de sus funciones o conocimientos profesionales son acciones que dan signos de una confianza y actitud desfavorable para la construcción de la resiliencia en los docentes. Al menos ocho docentes $(72.7 \%)$ coinciden con este tipo de respuestas. Este resultado difiere de lo encontrado en la investigación de Díaz-Sánchez y Barra (2017), en la cual las características disposicionales en los escenarios complejos donde realizan su trabajo son positivas y están relacionadas con la resiliencia de los docentes.

Asimismo, el resultado del ítem 16 de la variable Habilidad de resolución de problemas da evidencia de que los nuevos retos y desafíos de la educación frecuentemente le ocasionan estrés a la mayoría de los docentes. De esta misma variable, el ítem 17 presenta un posicionamiento negativo en el $27.3 \%$ de los participantes, dando cuenta de que una parte del grupo (tres docentes) considera que tendrá contratiempos al atender los nuevos retos y desafíos de la educación. Contrariamente a lo aquí encontrado, en Flores-Espinoza (2017) se mostró que la alta motivación de los docentes para realizar su trabajo en condiciones adversas es percibida y transmitida a los estudiantes; además su confianza y seguridad (Yo puedo), al estar en óptimas condiciones, motivaron a los docentes a resolver los problemas que se enfrentaban; al mismo tiempo sus alumnos se sentían motivados a afrontar con actitud pertinente los nuevos retos y desafíos de la educación, debido a que observaban a sus profesores.

La variable Condiciones de salud y física obtuvo un ítem negativo (19), las enfermedades comunes son frecuentes en la mayoría de los docentes. Esto puede tener consecuencias en su desempeño, en sus actividades cotidianas y en la interacción con sus pares o estudiantes. Aunado a esto se encuentra que los ítems 18, 20, 21 y 22 tienen 
Tabla 2. Resultados por ítem.

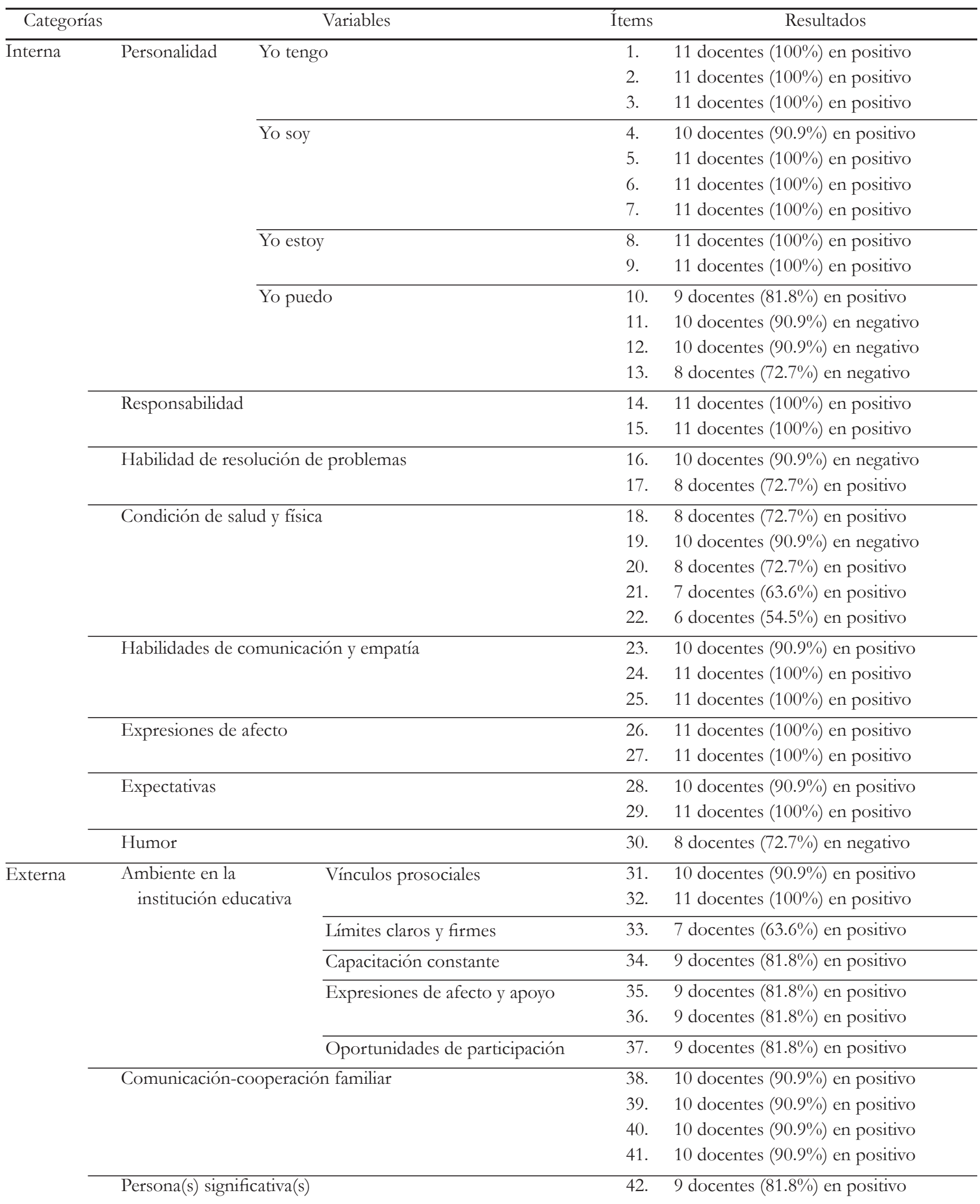


áreas de oportunidad con al menos el 25\% de los docentes. Los cuatro ítems dan cuenta de que algunos presentan enfermedades crónicas (tres docentes [27.3\%]), no tienen cuidados especiales con su alimentación (tres docentes [27.3\%]), no realizan alguna actividad deportiva (cuatro docentes [36.3\%]) y/o tienen sobrepeso (cinco docentes [45.4\%]), problemas que pueden estar relacionados con las enfermedades comunes. Este resultado coincide con la investigación de Cajigal, Maldonado y González-Gaudiano (2017), en la cual los docentes de nivel bachillerato que habían vivido situaciones adversas como las inundaciones en el estado de Veracruz, México, mostraron como área de oportunidad las Condiciones físicas y de salud. En dos contextos diferentes de país, el sobrepeso, el bajo cuidado en la alimentación y la poca actividad deportiva son problemas comunes en los docentes. Al vivir una adversidad se tienen más posibilidades de verse afectados si se encuentran en malas condiciones, de ahí la importancia de atender este factor.

Finalmente, en cuanto a la categoría Interna, la variable Humor resulta negativa (ítem 30). El humor es una estrategia que puede activar sus fortalezas ante las adversidades. Se ha visto que una característica que destaca en los docentes de nivel bachillerato resilientes es el humor (Cajigal, Maldonado y González-Gaudiano, 2017), no obstante lo aquí encontrado coincide con la investigación de Fontaines y Urdaneta (2009), en la cual el comportamiento de los docentes universitarios resilientes que participaron en su estudio no se caracterizaba por el buen humor.

Por otro lado, los resultados de la categoría Externa revelan que ninguna variable fue negativa. Solo el ítem 33 presenta un área de oportunidad con al menos el 25\% de los docentes; este pertenece a la variable Limites claros y firmes, refiere a la aplicación de reglas a todos los trabajadores de manera justa, por tanto, una parte del grupo de docentes no considera que esto exista, y su actuar puede mostrar insatisfacción al no reconocer la aplicación sin distinción de las reglas y políticas.

Continuando con la presentación de resultados, al efectuar las relaciones (con Chi cuadrada de Pearson) de la variable Antigüedad con los ítems se encontraron algunas significativas, es el caso del ítem 4 (0.048 Sig.), "Muchas personas me demuestran su aprecio constantemente"; los docentes con más años en la universidad (25 y 32 años) fueron los que se posicionaron de manera positiva, contrariamente a los de menor antigüedad (10 y 15 años), que manifestaron desacuerdo con la afirmación. Lo encontrado fue esperado, las relaciones sociales tienen más probabilidades de fortalecerse con el tiempo, de ahí que las personas con más antigüedad reciban muestras de aprecio constantemente. No obstante, este resultado da la oportunidad de voltear la atención hacia el resto de los docentes y propiciar un ambiente laboral en donde las muestras de aprecio sean para todos los sectores.

De igual forma, el ítem 21 tuvo relación significativa (0.043 Sig.), dando cuenta de que los de mayor ( 25 y 32 años) y menor antigüedad (10 y 15 años) son los que tienen una inconstante actividad deportiva, opuesto a los de mediana antigüedad (16, 
17, 18 años), que fueron los que más aportaron al resultado positivo. Este resultado complementa la información de la variable Condiciones físicas y de salud, que se muestra como un área de oportunidad y refuerza la propuesta, más adelante descrita, de involucrar a los docentes en actividades deportivas.

Continuando con las relaciones de los ítems, pero ahora con la variable Edad, se puede señalar que no se encontró relación significativa con alguno. En este tenor, la variable Género solo mostró tres relaciones significativas: el ítem 13, que afirma "Rechazo actividades porque no están relacionadas con mis conocimientos profesionales”, fue significativo con el género (0.028 Sig.) y se cargó de manera positiva más en las mujeres que en los hombres; dicho diferente, las mujeres no rechazan actividades solo por no estar dentro de sus conocimientos profesionales y los hombres sí lo hacen. Otra relación significativa (0.011 Sig.) con el género fue el ítem 17, "Los nuevos desafíos y retos de la educación los veo como un asunto que resolveré con algunos contratiempos", este resultado da cuenta de que los hombres son los que más aportan al resultado positivo. Visto de otra manera, las mujeres consideran que los nuevos retos y desafíos de la educación les pueden ocasionar dificultades cuando los solventen. Finalmente, el ítem 35, que afirma "Las expresiones de aprecio de mis estudiantes, colegas y/o superiores, son frecuentes", es significativo (0.035 Sig.) al relacionarlo con el género, dando cuenta de que son las mujeres las que más se posicionan en el lado positivo, es decir, son las que más expresiones de aprecio tienen por sus estudiantes y compañeros de trabajo. Estos resultados no son confrontados con otros hallazgos, pues su valor está en mostrar que la resiliencia es una construcción que varía en los sujetos, independientemente de su edad o género. La personalidad, el sentido de responsabilidad, la habilidad de resolución de problemas, las condiciones de salud y física, las habilidades de comunicación y empatía, las expresiones de afecto, expectativas, el humor, el ambiente en la institución, la cohesión familiar y las personas significativas, son factores que sí pueden incidir en la resiliencia de los docentes.

En definitiva, los resultados aquí presentados y el diálogo frente a otros hallazgos dan muestra de que no existe un punto de encuentro único en las investigaciones sobre la resiliencia de los docentes. Incluso, desde el punto de vista de Díaz-Sánchez y Barra (2017), suelen ser contradictorios los resultados en las investigaciones de este tipo. Comprensibles son las diferencias entre investigaciones, al pensar en la resiliencia como capacidades que se pueden crear o mejorar, asimismo esta no es una condición permanente, evoluciona a través del tiempo conforme las personas se desarrollan en lo social, ambiental, actitudinal, intelectual, emocional y personal (salud física y mental). De ahí los contrastes entre los grupos de docentes estudiados.

\section{Acciones propuestas}

A pesar de que los resultados presentados permiten señalar que los docentes que participaron en este estudio poseen la mayor parte de los factores que abonan a la 
resiliencia en positivo (85.8\% de los ítems) y se ubican en la resiliencia aceptable, también dejan ver que hay seis áreas de oportunidad (14.2\% de los ítems) en la mayoría de los docentes, y seis áreas de oportunidad para una parte del grupo estudiado (menos del 25\% de los docentes) que les impiden transitar a la resiliencia deseable. Teniendo en cuenta esto último, se proponen acciones que pueden contribuir en la mejora de los factores que resultaron negativos.

La variable Personalidad mostró que la confianza y la actitud son áreas de oportunidad en la mayoría de los docentes. De acuerdo con De la Cruz (2007), elementos como la confianza y la actitud de toda persona que interactúa en un ambiente de trabajo se relacionan con la motivación, y esta última se fortalece desde la comunicación. Si todos los trabajadores y dirigentes otorgan especial cuidado a la comunicación, se incrementan las posibilidades de tomar decisiones acertadas, no obstante "cuando los datos son incompletos, deficientes o inadecuados, afectan la motivación, la actitud, la satisfacción, el rendimiento de los empleados y todas las áreas de la organización" (De la Cruz, 2007, p. 92), de ahí que la propuesta sea proveer una capacitación sobre la comunicación asertiva y eficiente, por profesionales en el área, a los diversos colaboradores de la Facultad.

Otra área por atender es la referente a la variable Condición de saludy física. Es preciso recordar que esta investigación es parte de una estrategia que pretende reducir el porcentaje de reprobación y deserción escolar, además de incrementar el desempeño escolar de estudiantes con el desarrollo de la resiliencia desde el programa de tutoría. En otros estudios (Cajigal et al., 2020) se identificó que una muestra representativa de jóvenes universitarios tuvo como área de oportunidad la misma variable que ahora resulta con docentes de nivel superior. En atención a los problemas sobre la condición física y de salud de los estudiantes, se establecieron actividades dentro del programa de tutorías. Específicamente se gestionaron los recursos de la misma universidad para que los estudiantes tuvieran asesorías de nutrición, activación física, atención psicológica y revisión médica. Los resultados fueron favorables: durante el semestre que se implementaron las actividades bajaron los porcentajes de reprobación y de deserción escolar. Por tanto, se sugiere gestionar los mismo recursos y actividades para los docentes.

Al mejorar las condiciones de salud y física de los docentes se puede mejorar su sentido del bumor, variable que también es área de oportunidad en la mayoría de los docentes. Desde la posición de Carbelo y Jáuregui (2006), aunque no existe suficiente investigación para señalar con objetividad que hay una relación con la salud y el buen sentido del humor, sí existe una percepción subjetiva de dicha relación. Estos mismos autores señalan que la idea de que el humor es saludable o de que existe un mejor humor a partir de una mejor salud, es popular y fomenta "un mayor bienestar y disfrute de la vida, e incluso (...) el crecimiento hacia una mayor humanidad y plenitud" (Carbelo 
y Jáuregui, 2006, p. 27), de ahí que lo propuesto en el párrafo anterior también puede atender la variable Humor.

Por último, la variable Limites claros y firmes también es un área de oportunidad para una parte del grupo (cuatro docentes [36.4\%]). Se considera que una actividad que puede contribuir a mejorar esta variable es la de socializar constantemente las reglas, compromisos, derechos y obligaciones de los docentes, así como compartir información sobre los procesos administrativos que son parte de sus actividades. En este sentido, también se considera importante fortalecer la percepción de la figura y el quehacer docente en ellos mismos. En la investigación de Díaz-Sánchez y Barra (2017), a pesar de que los docentes laboraban en escenarios complejos, se encontró relación positiva entre la resiliencia y el desempeño laboral, debido a que los docentes presentaban características de disposición y satisfacción por la actividad profesional que realizaban, por tanto, difundir la información sobre las reglas y motivar la satisfacción y la convicción por la docencia puede contribuir en esta variable que resulta negativa.

\section{Conclusiones}

Al concebir la tutoría universitaria como un programa integral, el trabajo que ahí se lleva a cabo no se limita a la atención de aspectos académicos y cognitivos, sino que considera elementos intelectuales, psicológicos, emocionales y personales. La tutoría, desde esta mirada, puede contribuir en la disminución de la deserción y reprobación (Faroa, 2017), no obstante, se deben generar propuestas para efectuar una tutoría integral.

En este sentido, se ha visto coincidencia en el desarrollo de la resiliencia con la tutoría. La resiliencia es una capacidad emocional que surge para resistir, recuperarse o transformarse ante las adversidades, y son aspectos como autoestima, confianza, personalidad, apoyo, figuras significativas, cohesión familiar, ambiente en la escuela, así como salud física y emocional, los que abonan en la construcción de personas resilientes. Los mencionados aspectos son parte de la formación que se pretende desde la tutoría integral.

Iniciales son los esfuerzos desde el programa de tutorías para desarrollar la resiliencia en jóvenes universitarios de la licenciatura de Educación de la UNACAR. En estos esfuerzos se han obtenido resultados positivos (Cajigal et al., 2020), sin embargo se identificaron áreas de oportunidad que deben ser atendidas. Para ello se vio pertinente indagar sobre el estado que guardan los factores resilientes de una figura significativa en los estudiantes: los docentes.

En específico, esta investigación conoció el estado que guarda cada uno de los aspectos que componen la resiliencia de los docentes de la LE de la UNACAR y propuso acciones para contribuir en la mejora de los factores que resultaron negativos. De manera puntual, se encontró 
que los docentes poseen elementos de resiliencia aceptables, mas no son los deseables, de ahí que las acciones propuestas están en torno a evidenciar la necesidad de capacitar en cuanto a la comunicación asertiva y eficiente para mejorar la confianza y la actitud; asimismo se espera que los docentes reciban asesorías sobre nutrición, activación física, revisiones médicas y atención psicológica, todo ello para apoyar condiciones físicas y de salud; finalmente se desea que las reglas, compromisos, derechos y obligaciones, así como los procesos administrativos que son parte de las actividades docentes, sean socializados de forma constante, además de apoyarles en su satisfacción y convicción laboral.

Los docentes resilientes tienen mayores oportunidades de participar pertinentemente en el desarrollo o mejora de la resiliencia de los estudiantes. La tutoría es un buen espacio para el desarrollo de la resiliencia de los estudiantes, desde ahí se pueden fortalecer cada uno de los elementos que abonan en la construcción de personas resilientes. Los estudiantes con características resilientes pueden enfrentar sus adversidades de mejor manera, por lo tanto, la reprobación y deserción escolar son problemáticas que se pueden atender desde la tutoría y bajo la teoría de la resiliencia.

Con base en el trabajo realizado y en los resultados obtenidos se pueden plantear las siguientes conclusiones: 1).- Los docentes que laboran en el programa de educación de la UNACAR poseen la mayoría de factores en un estado positivo $(85.8 \%$ de los ítems), de ahí que pueden considerarse con resiliencia aceptable. 2).- Existen áreas de oportunidad en los factores que inciden en la resiliencia de los docentes, que pueden atenderse con los mismos recursos y actividades que ya están establecidos en el programa de tutorías de los estudiantes; solo es necesario que participen, que acompañen y se involucren en las acciones de tutoría. De igual forma se presentan áreas de oportunidad que pueden mejorarse cuando la comunicación sea asertiva y efectiva. Finalmente, se ve necesario fortalecer la percepción de la figura docente y su quehacer, así como dejar claras las reglas y compromisos que tienen en la universidad. 3).- $\mathrm{Al}$ atender las áreas de oportunidad detectadas, la resiliencia de los docentes puede incrementarse y lograrán participar de mejor manera en la construcción de la resiliencia de sus estudiantes.

\section{REFERENCIAS}

Acevedo, V. E., y Restrepo, L. (2012). De profesores, familias y estudiantes: fortalecimiento de la resiliencia en la escuela. Revista Latinoamericana de Ciencias Sociales, Niñezy Juventud, 10(1), 301-319.

Aguaded, G., y Almeida, N. A. (2016). La resiliencia del docente como factor crucial para superar las adversidades en una sociedad de cambios. Tendencias Pedagógicas, 58(2), 167-180.
Cajigal, E., Hernández-Marín, G., Yon, S., y Arias, L. (2020). Resiliencia de tutorados. Un caso de la Facultad de Ciencias Educativas de la Universidad Autónoma del Carmen, México. Revista Formación Universitaria, 13(2), 39-52. http://dx.doi.org/10.4067/ S0718-50062020000200039.

Cajigal, E., Maldonado, A. L., y González-Gaudiano, E. J. (2017). Resiliencia en docentes: una vía para 
mejorar capacidades de las poblaciones ante inundaciones agravadas por el cambio climático. Rev. Diálogo Educ., Curitiba, 17(55), 1445-1464. http://dx.doi. org/10.7213/1981-416X.17.055.DS01.

Carbelo, B., y Jáuregui, E. (2006). Emociones positivas: humor positivo. Papeles del Psicólogo, 27(1), 18-30.

Cruz-Flores, G., Cheyhaybar, E., y Abreu, L. F. (2011). Tutoría en educación superior: una revisión analítica de la literatura. Revista de la Educación Superior, 60(157), 190-209.

De la Cruz, N. (2007). La motivación, comunicación y actitudes de los empleados como elementos fundamentales en la organización. Perspectivas Psicológicas, 91-95.

Díaz-Sánchez, C., y Barra, E. (2017). Resiliencia y satisfacción laboral en profesores de colegios municipales y particulares subvencionados de la comuna de $\mathrm{Ma}-$ chalí. Estudios Pedagógicos, 43(1), 75-86. http:/ /dx.doi. org/10.4067/S0718-07052017000100005.

Faroa, B. D. (2017). Considering the role of tutoring in student engagement: Reflections from a South African university. Journal of Student Affairs in Africa, 5(2), 1-15.

Flores-Espinoza, M. R. (2017). Motivación y resiliencia en el docente. Fortaleza y oportunidad para la escuela en El Salvador. Revista Latinoamericana de Estudios Educativos, 47(3-4), 141-154.

Fontaines, T., y Urdaneta, G. (2009). Aptitud resiliente de los docentes en ambientes universitarios. Revista de Artes y Humanidades UNICA, 10(1), 163-180.

Guerra-Martín, M. D., Lima-Serrano, M., y LimaRodríguez, J. S. (2017). Effectiveness of tutoring to improve academic performance in nursing students at the University of Seville. Journal of New Approaches In Educational Research, 6(2), 93-102. https://doi. org/10.7821/naer.2017.7.201.

Henderson, N. (2001). Introducción. Nuevas tendencias en resiliencia. En A. Melillo y E. N. Suárez Ojeda (comps.), Resiliencia descubriendo las propias fortalezas (pp. 19-30). Argentina: Paidós.
Henderson, N., y Milstein, M. (2003). Resiliencia en la escuela. Argentina: Paidós.

Jadue, G., Galindo, A., y Navarro, L. (2005). Factores protectores y factores de riesgo para el desarrollo de la resiliencia encontrados en una comunidad educativa en riesgo social. Estudios Pedagógicos, 31(2), 43-55.

López-Roldán, S. F. (2016). Metodología de la investigación social cuantitativa. España: Universidad Autónoma de Barcelona.

Melillo, A. C. (2001). Prefacio. En A. Melillo y E. Suarez Ojeda (comps.), Resiliencia descubriendo las propias fortalezas (pp. 15-18). Argentina: Paidós.

Morán-Astorga, M. C., Finez-Silva, M. J., Menezes dos Anjos, E., Pérez-Lancho, M. C., Urchaga-Litago, J. D., y Vallejo-Pérez, G. (2019). Estrategias de afrontamiento que predicen mayor resiliencia. International Journal of Developmental and Educational Psychology, 4(1), 1-8. https://doi.org/10.17060/ijodaep.2019.n1.v4.1542.

Narro, J., y Arredondo, M. (2013). La tutoría. Un proceso fundamental en la formación de los estudiantes universitarios. Perfiles Educativos, 35(141), 132-151.

Noriega, G., Angulo, B., y Angulo, G. (2015). La resiliencia en la educación, la escuela y la vida. Perspectivas docentes, $58(1), 42-48$.

Ortega-González, Z., y Mijares, B. (2018). Concepto de resiliencia: desde la diferenciación de otros constructos, escuelas y enfoques. Revista Científica Electrónica de Ciencias Humanas, 39(13), 30-43.

Richardson, G. E., Neiger, B. L., Jensen, S., y Kumpfer, K. L. (1990). The resiliency model. Health and Education, 21(6), 33-39.

Taormina, R. J. (2015). Adult personal resilience: A new theory, new measure, and practical implications. Psychological Thought, 8(1), 35-46.

Taber, K. S. (2017). The use of Cronbach's Alpha when developing and reporting research instruments in science education. Res Sci Educ, 48(1), 1273-1296. DOI 10.1007/s11165-016-9602-2.

Cómo citar este artículo:

Cajigal Molina, E. (2021). Docentes resilientes. Elementos centrales en el programa universitario de tutoría. IE Revista de Investigación Educativa de la REDIECH, 12, e1115. doi: 10.33010/ie_rie_rediech.v12i0.1115. 